\title{
Effect of neoadjuvant chemotherapy on locally advanced upper tract urothelial carcinoma: a pooled analysis
}

\author{
Dongxu Qiu", Jiao Hu\#, Tongchen He, Huihuang Li, Jian Hu, Zhenglin Yi, Jinbo Chen, Xiongbing Zu \\ Department of Urology, Xiangya Hospital, Central South University, Changsha, China \\ Contributions: (I) Conception and design: D Qiu; J Hu; (II) Administrative support: X Zu; J Chen; (III) Provision of study materials or patients: J Hu; \\ Z Yi; (IV) Collection and assembly of data: H Li; T He; (V) Data analysis and interpretation: D Qiu; J Hu; (VI) Manuscript writing: All authors; (VII) \\ Final approval of manuscript: All authors. \\ "These authors contributed equally to this work. \\ Correspondence to: Xiongbing Zu; Jinbo Chen. Department of Urology, Xiangya Hospital, Central South University, Changsha 410008, China. \\ Email: zuxbxyyy@126.com; chenjinbo1989@yahoo.com.
}

Background: The outcome of neoadjuvant chemotherapy (NAC) has been established in bladder cancer but remains controversial in upper tract urothelial carcinoma (UTUC). In this work, we explored the therapeutic effect of NAC in patients with locally advanced UTUC.

Methods: We conducted a literature search on articles published from 1995 up to April 2020 in PubMed/ Medline, the Cochrane Library, Embase, Google Scholar. A total of 19 eligible studies with 6,283 patients were identified, from which the overall survival (OS), cancer-specific survival (CSS), progression-free survival (PFS), disease-free survival (DFS), pathological complete response (pCR) rate and pathological partial response (pPR) rate were extracted. All analyses were conducted using Review Manager 5.3 and Stata statistical software (version 15$)$.

Results: In total, 6,283 UTUC patients were included from 19 eligible studies out of which 1,474 patients received NAC and subsequent radical nephroureterectomy (RNU), whereas 4,809 patients received RNU only. Compared with single RNU, patients with NAC and subsequent RNU exhibited longer OS, CSS, PFS, DFS by hazard ratio (HR) 2.14 [95\% confidence interval (CI): 1.75-2.63; P<0.001], HR 2.07 (95\% CI: 1.49-2.87; P<0.001), HR 2.00 (95\% CI: 1.42-2.83; P<0.001), and HR 3.76 (95\% CI: 2.16-6.56; $\mathrm{P}<0.001$ ). pCR rate and pPR rate of NAC are $0.10(0.07-0.13)$ and 0.40 (95\% CI: $0.32-0.49, \mathrm{P}<0.001)$ respectively.

Conclusions: This work revealed that NAC and subsequent RNU provided better survival outcomes in patients with locally advanced UTUC when compared with single RNU.

Keywords: Upper tract urothelial carcinoma (UTUC); neoadjuvant chemotherapy (NAC); survival outcomes; effect; pooled analysis

Submitted May 18, 2020. Accepted for publication Sep 14, 2020.

doi: $10.21037 /$ tau-20-933

View this article at: http://dx.doi.org/10.21037/tau-20-933

\section{Introduction}

Upper tract urothelial carcinoma (UTUC) is a relatively rare disease and the proportion is only $5-10 \%$ in urothelial carcinomas, about 2 cases in 100,000 residents in Western countries $(1,2)$. Overall, compared with $15-25 \%$ cases of bladder tumors, $60 \%$ of UTUCs are invasive diagnosis $(1,3)$. Following the EAU guideline, UTUC is recommended in radical nephroureterectomy (RNU) with excision of ipsilateral bladder cuff (1), however, high recurrence rate of advanced UTUC after standard has been reported to cause an unsatisfying prognosis and inaccurate treatment $(3,4)$. Fortunately, increasing assessments have revealed that both neoadjuvant chemotherapy (NAC) and adjuvant chemotherapy (AC) may improve the survival in advanced UTUC $(5,6)$. 
Moreover, the effect of NAC in advanced bladder cancer has been affirmed through randomized clinical trials (7). Due to the infrequency of UTUC, clinical trials of NAC in UTUC may be associated with drawbacks, among them, difficulty in enrollment and longtime follow-up. A published prospective study that enrolled 30 patients revealed pathological complete response (pCR) without any prognosis indicators (8). Several retrospective studies have also confirmed high overall survival (OS) and disease-free survival (DFS) by examining the influence of perioperative chemotherapy for UTUCs. Compared with AC, NAC may offer additional benefits. For example, for patients whose renal function is damaged by RNU, chemotherapy or higher doses of chemotherapy may be unsuitable compared with NAC (9-11). A phase 3, open-label, randomized controlled trial with 126 participants showed that AC significantly improved DFS (HR 0.45, 95\% CI: 0.30-0.68; $\mathrm{P}=0.0001)$ (12), but $44 \%$ patients developed acute grade 3 or worse treatment-emergent adverse events $(\mathrm{P}<0.0001)$. Additionally, some UTUC patients are excluded from AC because of insufficient recovery after surgery, this might underestimate the incidence rate of adverse events. By 1995, Igawa et al. began to adopt cisplatin-based NAC to manage 15 advanced UTUC patients. Notably, the patients showed a $13 \%$ pathologic complete response (pCR) rate, a $40 \%$ pathological partial response (pPR) rate, and an overall response rate of $53 \%$ (13). Several studies also proved that NAC potentially exerted pPR and pCR. Besides, numerous investigations demonstrated that NAC provided better survival outcomes than when surgery is used alone (14). On the contrary, other studies found no differences in survival outcomes when they compared patients who underwent NAC plus RNU with those subjected to surgery without NAC $(15,16)$. Therefore, the treatment efficacy of NAC in advanced UTUC remains elusive, and whether NAC could serve as a more suitable management tool deserves further in-depth studies.

Recently findings published by Kim and his colleagues that investigated the effect of NAC on locally advanced UTUC patients indicate that patients subjected to NAC plus RNU showed better survival outcomes (17). But they pooled only 4 studies in their analysis, which rendered their results inaccurate and incomplete. This work, therefore, purposed to provide a more comprehensive and up to date report that evaluates the efficacy of NAC in advanced UTUC patients. We present this article in accordance with the PRISMA reporting checklist (available at http:// dx. doi. org/10. 21037/tau-20-933).

\section{Methods}

\section{Search strategy}

All the related articles were identified from PubMed, Embase, Cochrane Library, and Google Scholar until April 2020. The selection criterion is highlighted in Figure 1. The search terms included: (((neoadjuvant chemotherapy) OR (perioperative chemotherapy) OR (preoperative chemotherapy))) AND ((upper tract urothelial carcinoma) OR (Ureteral Neoplasms) OR (kidney pelvis carcinoma)) AND ((Prognosis) OR (Prognostic Factors) OR (Prognostic Factor) OR (Factors, Prognostic)). To complete the search, these terms were either searched separately or in a combinational manner. All identified studies were reviewed, original studies listed as references, and examined through a manual search by different authors, independently.

\section{Inclusion and exclusion criteria}

A total of 19 articles were selected based on the following 5 criteria (PICOS principle): (I) population (P), patients pathologically diagnosed as advanced UTUC; (II) intervention (I): treated with NAC with subsequent RNU; (III) comparison (C): treated with RUN only; (IV) outcomes (O): prognosis indicators including OS (periods from the start of treatment to death from any cause), cancer-specific survival (CSS) (cancer survival in the absence of other causes of death), progression-free survival (PFS) (periods from the start of treatment to disease progression or death from any cause), DFS (periods from the start of treatment to disease recurrence or death from any cause), pCR rate (achieve pT0N0 disease condition after treatment) and pPR rate (achieve $\leq \mathrm{pT} 2 \mathrm{~N} 0$ disease condition after treatment); (V) study design (S), both randomized controlled trials and retrospective trials relative to this subject. Eligible studies were identified as follows: (I) accurately defines prognosis indicators. (II), Have data in pathological response rate, the hazard ratio (HR) with $95 \%$ confidence interval (CIs), or sufficient original data to calculate pathological response rate or HR and 95\% CI. (III) Patients pathologically diagnosed as advanced UTUC. (IV) Reliable quality evaluated by Grading of Recommendations Assessment, Development, and Evaluation (GRADE) system. (V) Published article in English language on a human subject. Studies on bladder urothelial cancer, UTUC treated with AC and unsuitable forms such as reviews, case series, case reports, editorials, letters, among others were excluded. 


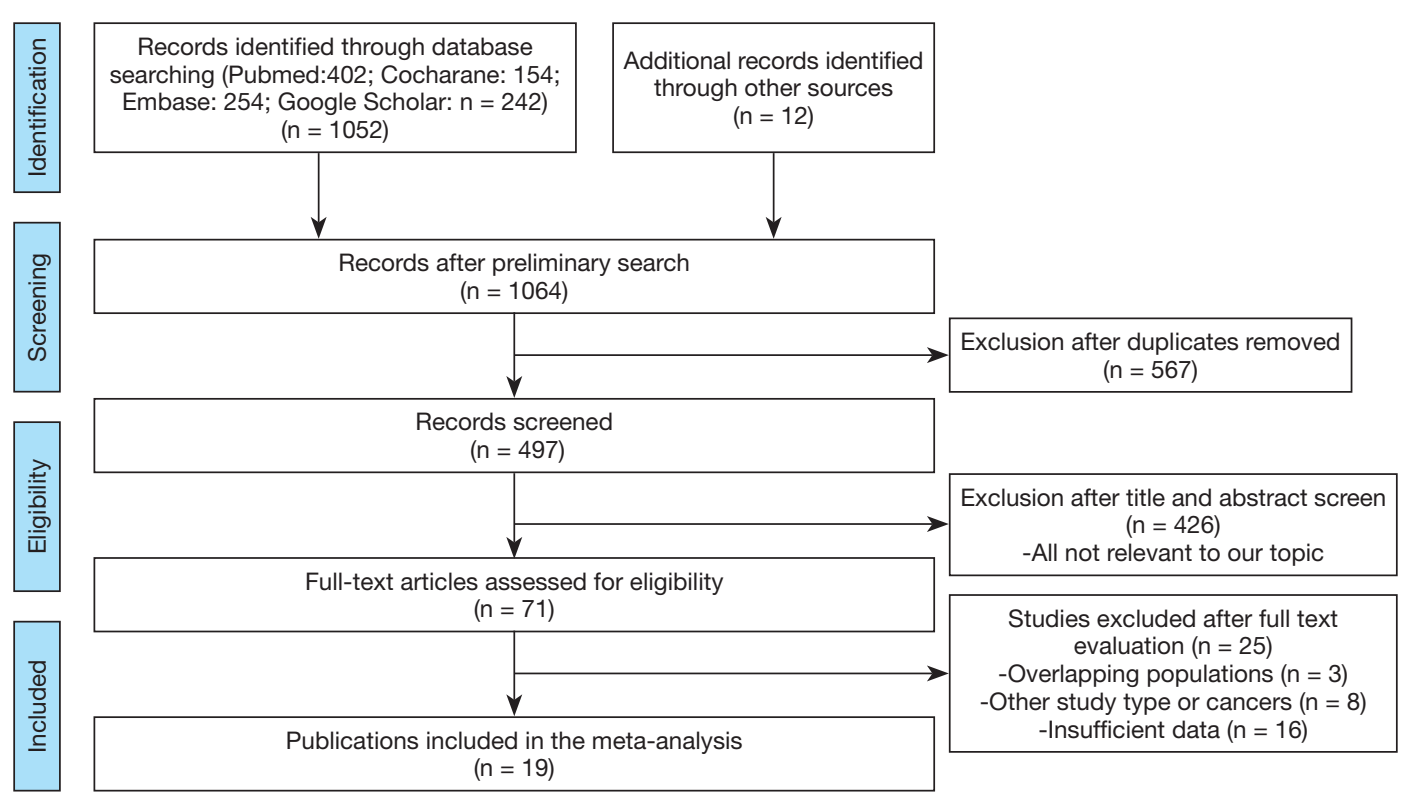

Figure 1 Flowchart for article selection.

\section{Data extraction and quality assessment}

In extracting data from the enrolled studies, 3 authors (Dongxu Qiu, Jiao Hu, and Tongchen He) reviewed each article and independently collected data from the 19 published studies. Any emerging conflict was resolved through debate. Extracted items from those articles included author, year, country, study design, tumor stage, NAC regimen, and oncologic outcomes (OS, CSS, PFS, DFS, pCR, pPR). The effective percentage of available HRs and $95 \%$ CIs provided by articles were directly extracted. Then, using originally recorded statistical data, the HRs and $95 \%$ CIs were calculated.

The quality of the articles was evaluated by 3 authors (Dongxu Qiu, Jiao Hu, and Tongchen He) using methodology, precision of results, consistency of results, directness, and risk of publication bias according to GRADE system. As a result, all studies were classified into one out of 4 evidence quality levels (high, moderate, low, and very low). In addition, publication bias was evaluated through visual inspection of funnel plots, whereas to determine the reliability of each result, a sensitivity analysis was conducted using the leave-one-out cross-validation

\section{Statistical analysis}

HRs, 95\% CIs of OS, CSS, PFS, and DFS were extracted or calculated from enrolled studies. The $\mathrm{I}^{2}$ test estimated study variance. If the $\mathrm{I}^{2}<50 \%$, a fixed-effect model would be used, If the $\mathrm{I}^{2}>50 \%$, a random effect model would be applied. Publication bias was evaluated by funnel plots. As mentioned above, a sensitivity analysis was performed to assess the stability of each result. All the analyses were performed with Review Manager version 5.3 (The Nordic Cochrane Centre, The Cochrane Collaboration, Copenhagen) (including OS, PFS, DFS, CSS) and Stata statistical software (version 15) (including pCR and pPR rate). All $\mathrm{P}$-values were two-sided, and $\mathrm{P}<0.05$ was considered statistically significant.

\section{Results}

\section{Search results}

In total, 19 eligible studies were enrolled in the pooled analysis from which a total of 6,283 UTUC patients were recruited. Among them, 1,474 UTUC patients accepted NAC and subsequent RNU, while 4,809 patients underwent RUN only. The process of enrolling studies was documented in Figure 1. Extracted information for each enrolled study is presented in Table 1. The outcomes of each included study are provided in Table S1. The final pooled results of all studies are displayed in Table 2. 


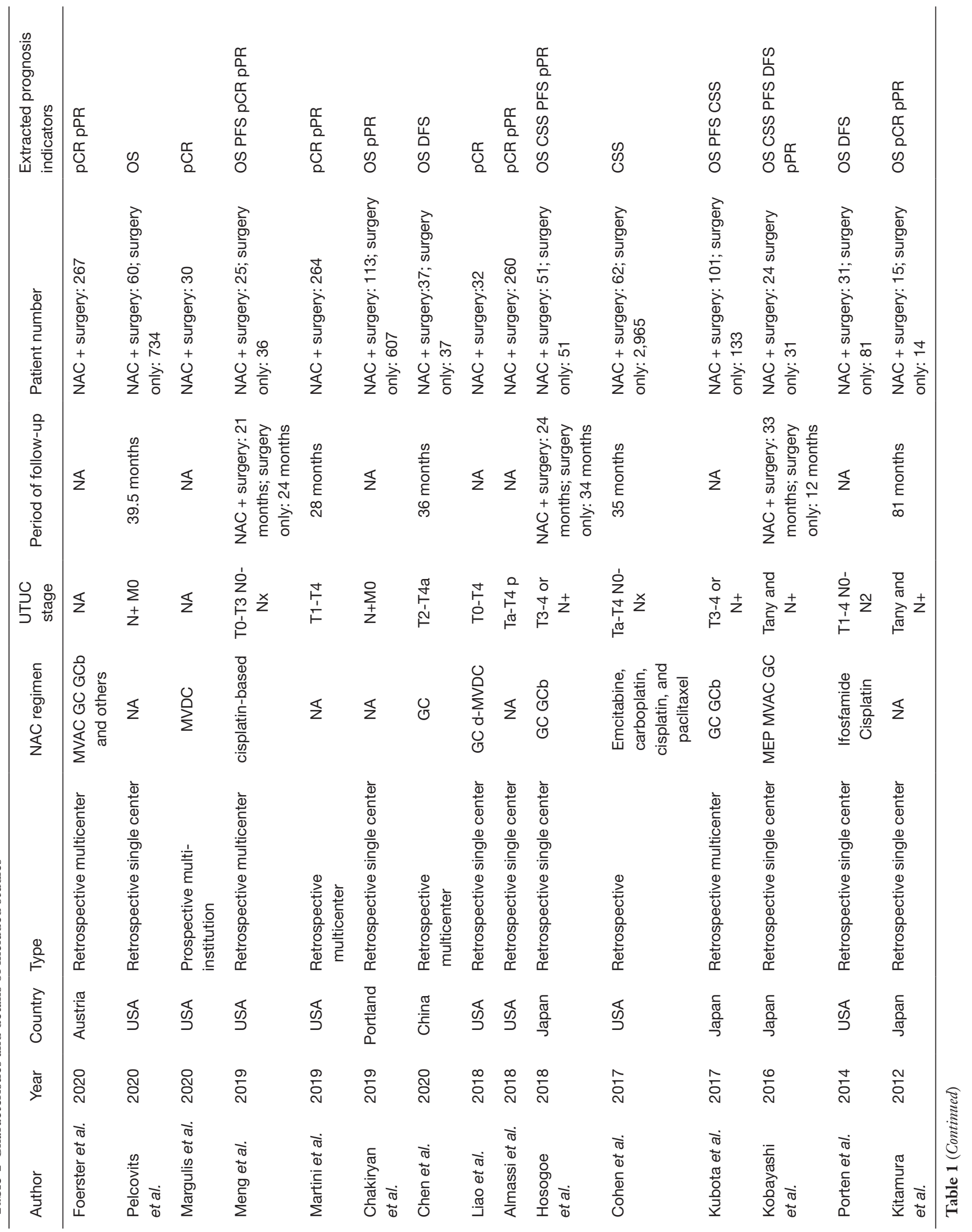




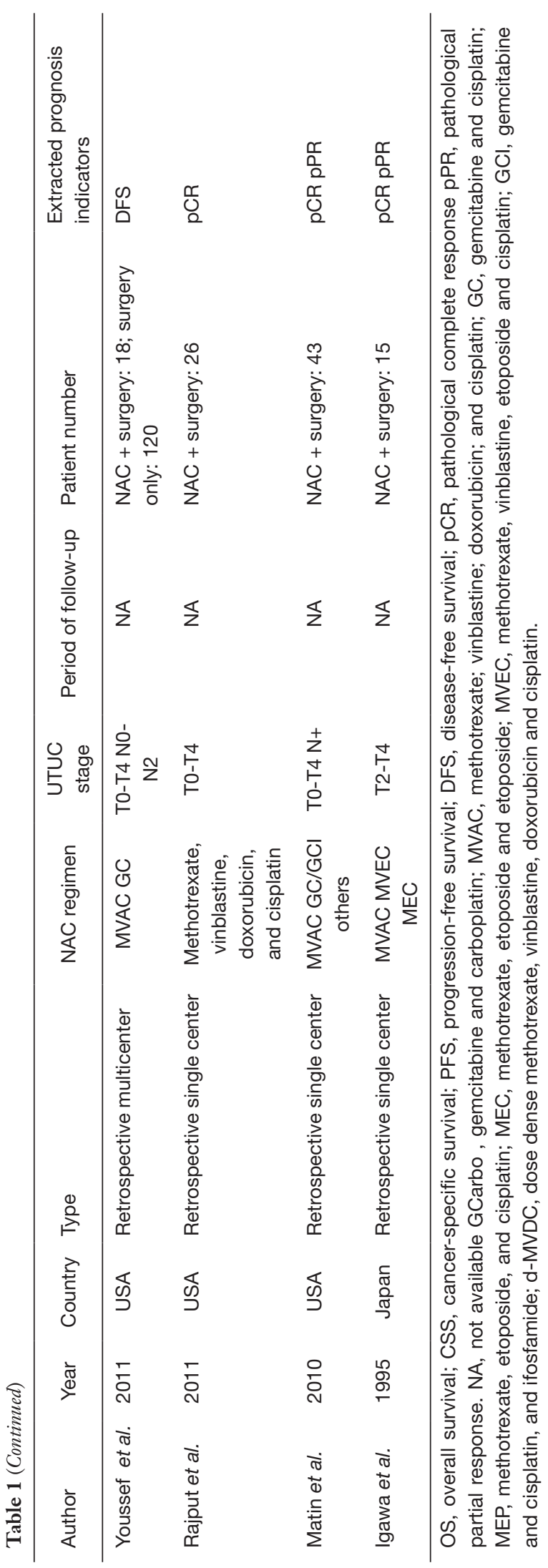

\section{OS}

In total, 9 included studies reported the OS results (14,18-25). After pooling the data across the 9 studies, we obtained the results were HR 2.14 (95\% CI: 1.75-2.63; $\mathrm{P}<0.001)$, which represented a 2.14 times OS benefit in UTUC patients undergoing NAC following surgery compared to surgery alone (Figure 2A). Notably, there was no significant heterogeneity among studies based on Cochran Q statistics $(\mathrm{P}<0.001)$ and $\mathrm{I}^{2}=8 \%$. Heterogeneity $\left(\mathrm{I}^{2}<50 \%\right)$ was low in OS analyses. Hence, this study used a fixed-effect model.

\section{CSS}

Four studies were pooled in the CSS subgroup (21-23,26). Pooled HR was calculated as 2.07 (95\% CI: 1.49-2.87; $\mathrm{P}<0.001)$, representing 2.07 times benefit in CSS (Figure 2B). Based on Cochran Q statistics $(\mathrm{P}<0.001)$, there was no heterogeneity among studies when $\mathrm{I}^{2}=0 \%$. Hence, we used a fixed-effect model.

\section{PFS}

PFS results were reported in 4 studies (19,21-23). After data were pooled across 4 studies, it was found that HR 2.00 (95\% CI: $1.42-2.83 ; \mathrm{P}<0.001$ ), which represents a 2 times PFS benefit in UTUC patients after using NAC following RNU compared with surgery alone (Figure $2 C$ ). No heterogeneity existed among studies based on Cochran $\mathrm{Q}$ statistics $(\mathrm{P}<0.001)$ and $\mathrm{I}^{2}=0 \%$. Heterogeneity $\left(\mathrm{I}^{2}<50 \%\right)$ was low in PFS analyses, thus, adopting a fixed-effect model.

\section{DFS}

Here, a total of 4 included studies were pooled in the DFS subgroup $(14,21,23,27)$. Pooled HR was calculated 3.76 (95\% CI: 2.16-6.56; $\mathrm{P}<0.001$ ), representing 3.76 times benefit in DFS (Figure 2D). Based on Cochran Q statistics $(\mathrm{P}<0.001)$, there was no heterogeneity among studies when $\mathrm{I}^{2}=0 \%$, therefore, we used a fixed-effect model.

\section{$p C R$ rate and $p P R$ rate}

pCR rate was reported in 10 studies $(8,13,16,19,25,28-32)$. After pooling data across the 10 studies, the pooled pCR rate was $11 \%$ (95\% CI: $0.07,0.14 ; \mathrm{P}=0.058)$, implying that about one in ten patients with UTUC treated with 
Table 2 Summary of pooled survival outcomes (NAC + surgery versus surgery alone in UTUC) and efficiency rate of NAC in UTUC

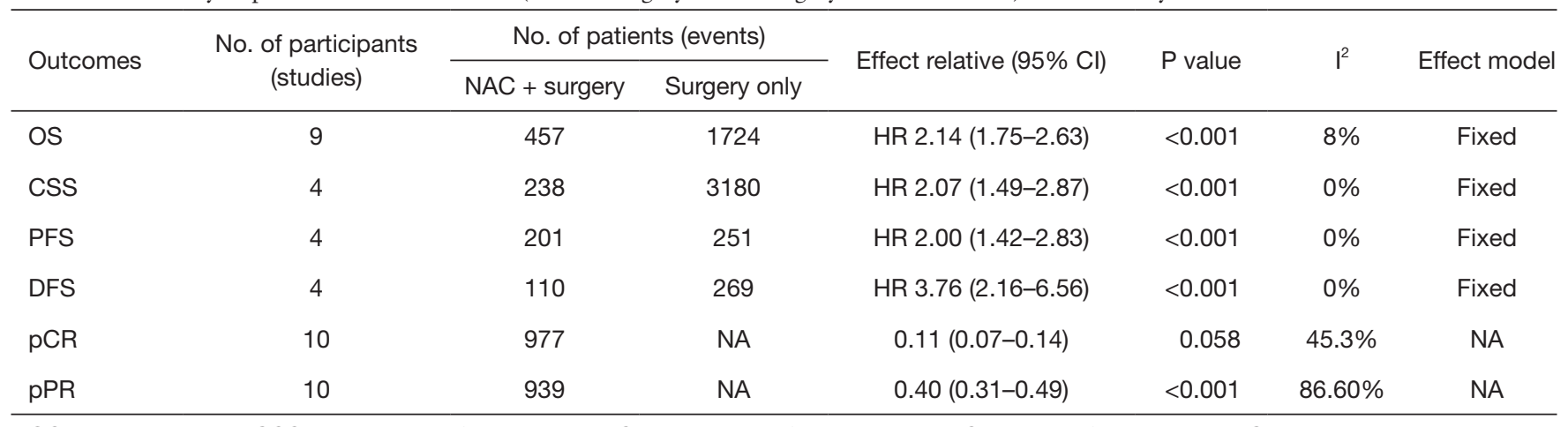

OS, overall survival; CSS, cancer-specific survival; PFS, progression-free survival; DFS, disease-free survival; pCR, pathological complete response; pPR, pathological partial response; NA, not available; $\mathrm{HR}$, hazard ratio; $\mathrm{Cl}$, confidence interval.

NAC can achieve pCR (Figure $3 A$ ). Heterogeneity existed among studies based on Cochran $\mathrm{Q}$ statistics $(\mathrm{P}=0.036)$ and $\mathrm{I}^{2}=34.7 \%$. In addition, $\mathrm{pPR}$ rate was displayed in 10 studies $(13,19-21,23,25,28,29,31,32)$. After analyzing data from the 10 studies, the pooled pPR rate was $40 \%$ ( $95 \%$ CI: 0.32-0.49; $\mathrm{P}<0.001)$, which revealed that about 4 in ten patients with UTUC treated with NAC can achieve pPR (Figure $3 B$ ). In subgroup analysis, pCR rate of patients with stage TxN0 was 0.11 (95\% CI: $0.05-0.16$; $\mathrm{P}=0.014$ ) (Figure $4 A$ ), and pCR rate of patients with stage $\mathrm{TxNx}$ was 0.12 (95\% CI: 0.03-0.21; $\mathrm{P}=0.200$ ) (Figure 4B). In subgroup analysis of $\mathrm{pPR}$ rate, patients with stage TxN0 was 0.27 (95\% CI: 0.23-0.31; $\mathrm{P}=0.339$ ) (Figure 4C), and patients with stage $\mathrm{TxNx}$ was 0.36 (95\% CI: $0.29-0.42 ; \mathrm{P}=0.520$ ) (Figure 4D).

\section{Quality assessment, sensitivity, and publication bias}

To evaluate how individual studies impacted the pooled results, a sensitivity analysis was performed by eliminating one study at a time. However, there were no significant changes thus, this verified the reliability of the pooled results. GRADE quality assessments of each outcome are shown in Table S2. The certainty of 4 comparisons and 2 response rates was extremely low. Funnel plots of each outcome are displayed in Figure S1.

\section{Discussion}

Urothelial cancers that transpire in the upper (including ureteral neoplasms or kidney pelvis carcinoma) or lower tracts (bladder cancer), exhibit similar biological, practical, anatomical features and other aspects, but also have some differences (33). At diagnosis, about $15 \%$ to $25 \%$ of bladder cancer cases are reported, whereas more than $60 \%$ of UTUC cases are diagnosed at advanced stages. This implies that UTUC is more invasive and with worse prognosis (9). The EAU guideline [2020] on UTUC recommends RNU plus excision of ipsilateral bladder cuff for high-grade UTUC (34). For patients with advanced UTUC, a higher prognosis benefit is achieved when chemotherapy and RNU are combined that when chemotherapy is used alone (35). With the recent advancement and increasing utilization of NAC in bladder cancer, increasing NAC utilization in highgrade UTUC aroused our interests. NAC utilization is currently more frequent compared to previous reports (32). It is worth noting that adjuvant therapy after RNU is restricted especially when there is decreasing renal function. In phase 3, open-label, randomized controlled trial (a POUT trial), better DFS outcome was reported in patients subjected to AC compared to the surveillance group (HR 0.45, 95\% CI: 0.30-0.68; $\mathrm{P}=0.0001$ ) (12). However, the side effects of AC could not be ignored at the same time. After AC, $44 \%$ of patients developed acute grade 3 or worse treatment-emergent adverse events, compared with $4 \%$ in the surveillance group $(\mathrm{P}<0.0001)$. Furthermore, some UTUC patients were excluded from the AC group due to the unsatisfactory recovery after RNU, which potentially underestimate the incidence rate of adverse events. On the other hand, NAC is not associated with a similar side effect and may play a more indispensable role in managing advanced UTUC (36,37). Elsewhere, a retrospective study reported no difference in prognosis between NAC plus RNU and RNU plus AC in high-grade UTUC patients, and the study hypothesized that patients who responded to NAC showed better survival compared with AC (38). 


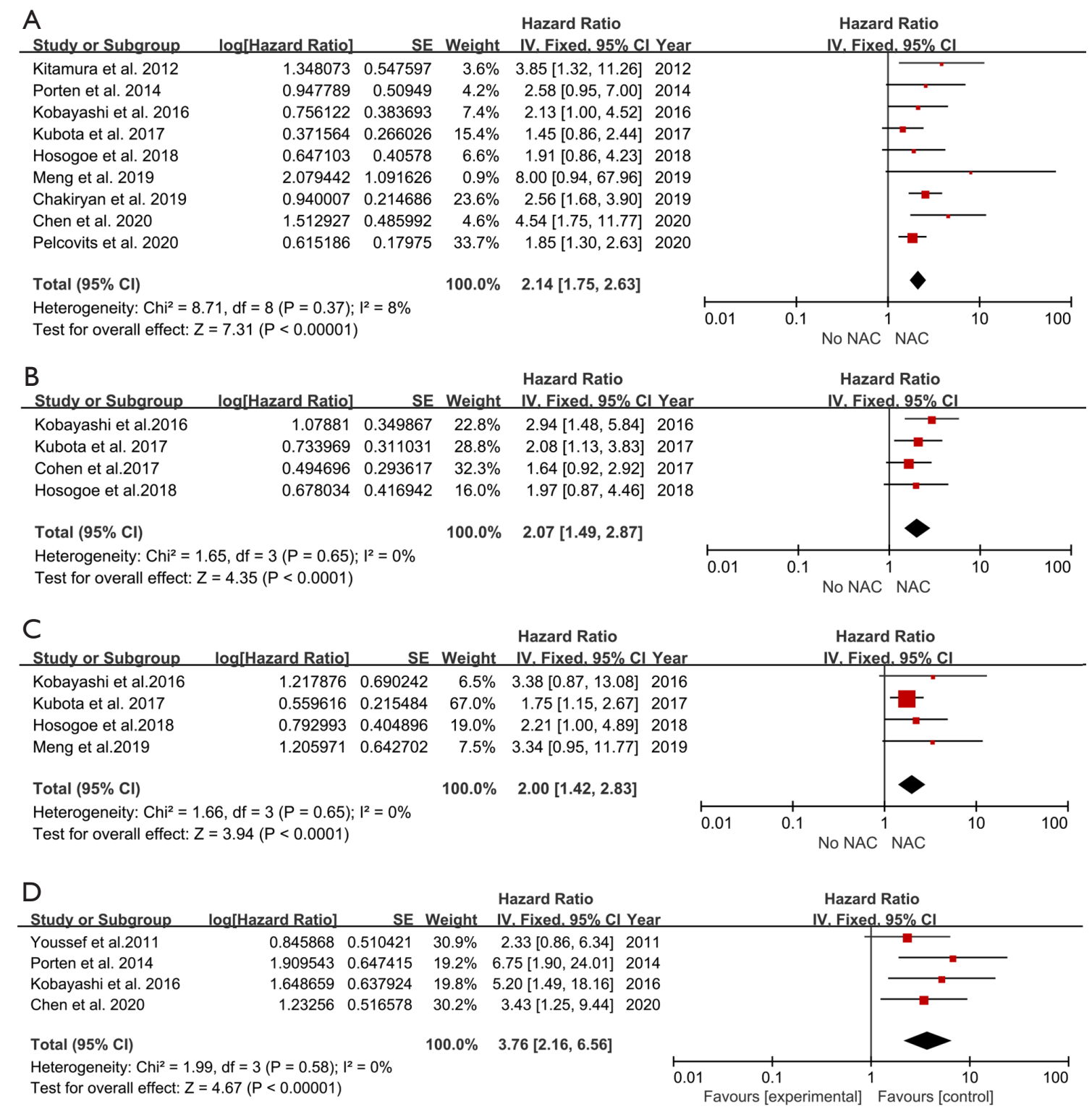

Figure 2 Pooled survival outcomes of UTUC patients treated with NAC plus RNU compared to RNU alone. (A) Overall survival (OS); (B) cancer-specific survival (CSS). (C) progression-free survival (PFS); (D) disease-free survival (DFS). UTUC, upper tract urothelial carcinoma; NAC, neoadjuvant chemotherapy; RNU, radical nephroureterectomy.

Whether NAC would achieve a better outcome than AC should be intensively explored. After reviewing published articles in PubMed/Medline, the Cochrane Library, Embase and Google Scholar, we identified most included articles to be retrospective. For keeping pace with research actuality of NAC in UTUC patients, we deeply analyzed about launching clinical trials about this subject. The recruitment status of NCT01663285 was terminated because it did not enroll enough participants. Besides, the recruitment status of NCT01261728 is active, not recruiting, without relevant published articles. Notably, one prospective article of NCT02412670 enrolled 30 patients in the study and showed pathologic complete response without other prognosis indicators (8). To ensure completeness and persuasiveness of our study, we included this article in the analysis. Other clinical trials such as NCT02876861, 


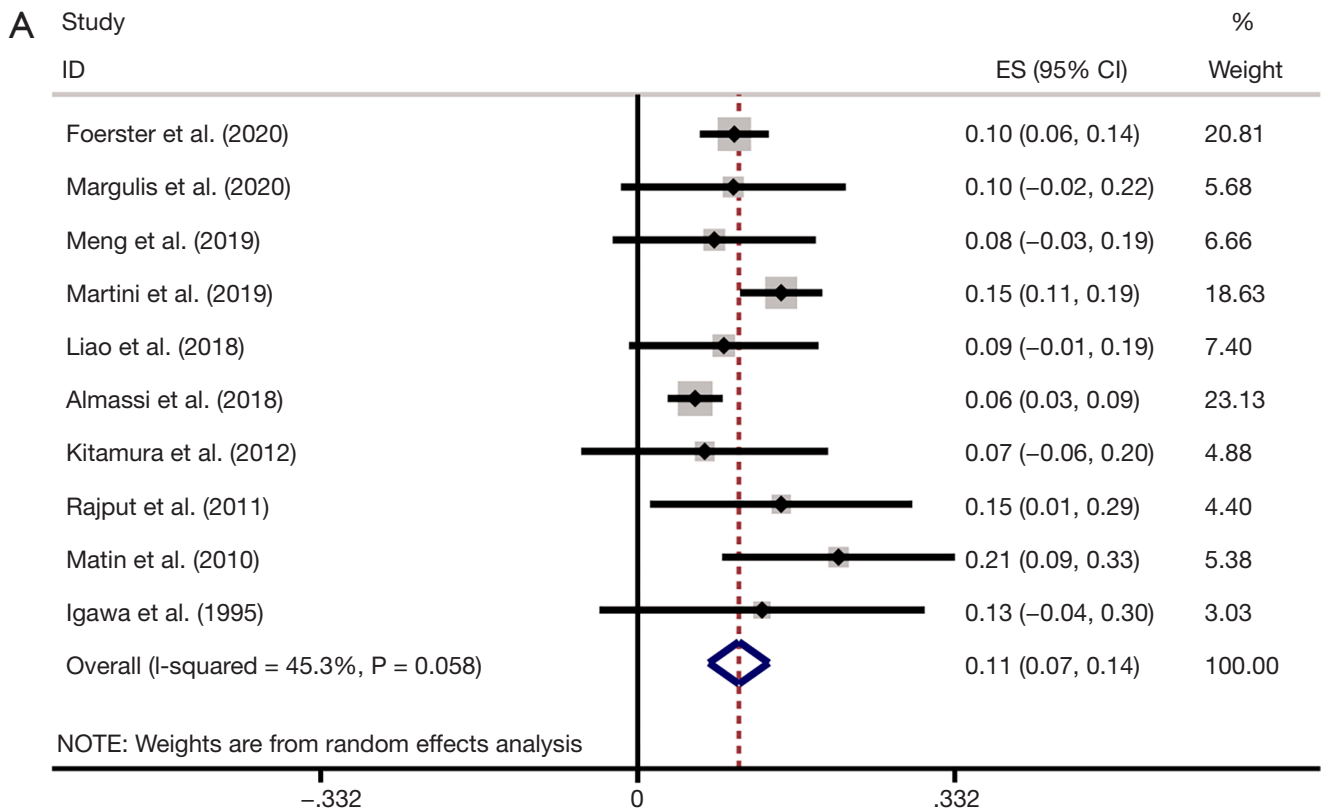

B Study

$\%$

\begin{tabular}{|c|c|c|}
\hline ID & ES $(95 \% \mathrm{Cl})$ & Weight \\
\hline Foerster et al. (2020) & $0.45(0.39,0.51)$ & 12.86 \\
\hline Martini et al. (2019) & $0.27(0.22,0.32)$ & 13.01 \\
\hline Meng et al. (2019) & $0.80(0.64,0.96)$ & 9.53 \\
\hline Chakiryan et al. (2019) & $0.33(0.24,0.41)$ & 12.07 \\
\hline Almassi et al. (2018) & $0.25(0.20,0.30)$ & 13.03 \\
\hline Kobayashi et al. (2015) & $0.38(0.18,0.57)$ & 8.22 \\
\hline Hosogoe et al. (2013) & $0.53(0.28,0.79)$ & 6.43 \\
\hline Kitamura et al. (2012) & $0.47(0.21,0.72)$ & 6.43 \\
\hline Matin et al. (2010) & $0.33(0.19,0.47)$ & 10.13 \\
\hline Igawa et al. (1995) & $0.40(0.21,0.59)$ & 8.28 \\
\hline Overall (I-squared $=86.6 \%, P=0.000$ ) & $0.40(0.32,0.49)$ & 100.00 \\
\hline NOTE: Weights are from random effects analysis & & \\
\hline
\end{tabular}

Figure 3 Pooled efficiency rates of UTUC patients treated with NAC. (A) Pathological complete response (pCR) rate; (B) pathological partial response (pPR) rate. UTUC, upper tract urothelial carcinoma; NAC, neoadjuvant chemotherapy.

NCT02969083 and NCT04099589 were conducted to assess the significance of NAC in UTUC, the status of 3 clinical trials is recruiting. The status of another clinical trial (NCT00696007) is withdrawn because the study did not recruit subjects meeting the inclusion criteria. We found no published article on the above 4 clinical trials. Additional details of clinical trials are displayed in the Table S3.
From the included studies, we revealed a smaller proportion of UTUC patients treated with NAC, but these patients exhibited a better prognosis compared to those who received surgery alone. After pooling data from 19 studies, we reported 2.14 times benefit in OS, 2.07 times benefit in CSS, 2 times benefit in PFS, and 3.76 times benefit in DFS, as well as, $10 \%$ pCR rate and $40 \%$ pPR 
A Study

ID

Martini et al. (2019)

Liao et al. (2018)

Almassi et al. (2018)

Rajput et al. (2011)

Iwaga et al. (1995)

Overall (I-squared $=68.1 \%, P=0.014)$

NOTE: Weights are from random effects analysis

$$
-.3
$$

B Study

ID

Meng et al. (2019)

Kitamura et al. (2012)

Matin et al. (2010)

Overall (I-squared $=37.8 \%, P=0.200)$

NOTE: Weights are from random effects analysis

$$
-.332
$$

C Study

ID

Martini et al. (2019)

Almassi et al. (2018)

Igawa et al. (1995)

Overall (I-squared $=7.5 \%, P=0.339)$

NOTE: Weights are from random effects analysis

$$
-.592
$$

$\%$

ES $(95 \% \mathrm{Cl}) \quad$ Weight

$0.15(0.11,0.19) \quad 30.11$

$0.09(-0.01,0.19) \quad 16.90$

$0.06(0.03,0.09) \quad 33.46$

$0.15(0.01,0.29) \quad 11.30$

$0.13(-0.04,0.30) \quad 8.23$

$0.11(0.05,0.16) \quad 100.00$

$\%$

ES $(95 \% \mathrm{Cl}) \quad$ Weight

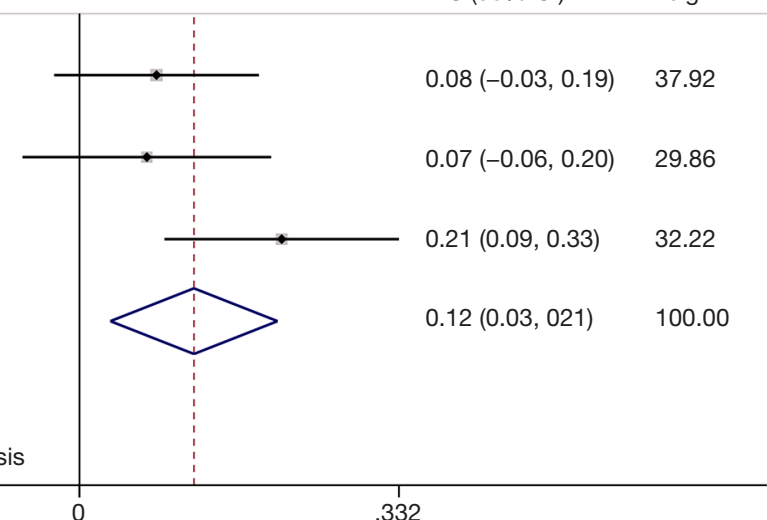

$\%$

Weight

ES $(95 \% \mathrm{Cl}) \quad$ Weight

$0.27(0.22,0.32) \quad 47.31$

$0.25(0.20,0.30) \quad 48.53$

$0.40(0.21,0.59) \quad 4.16$

$0.27(0.23,0.31) \quad 100.00$ 


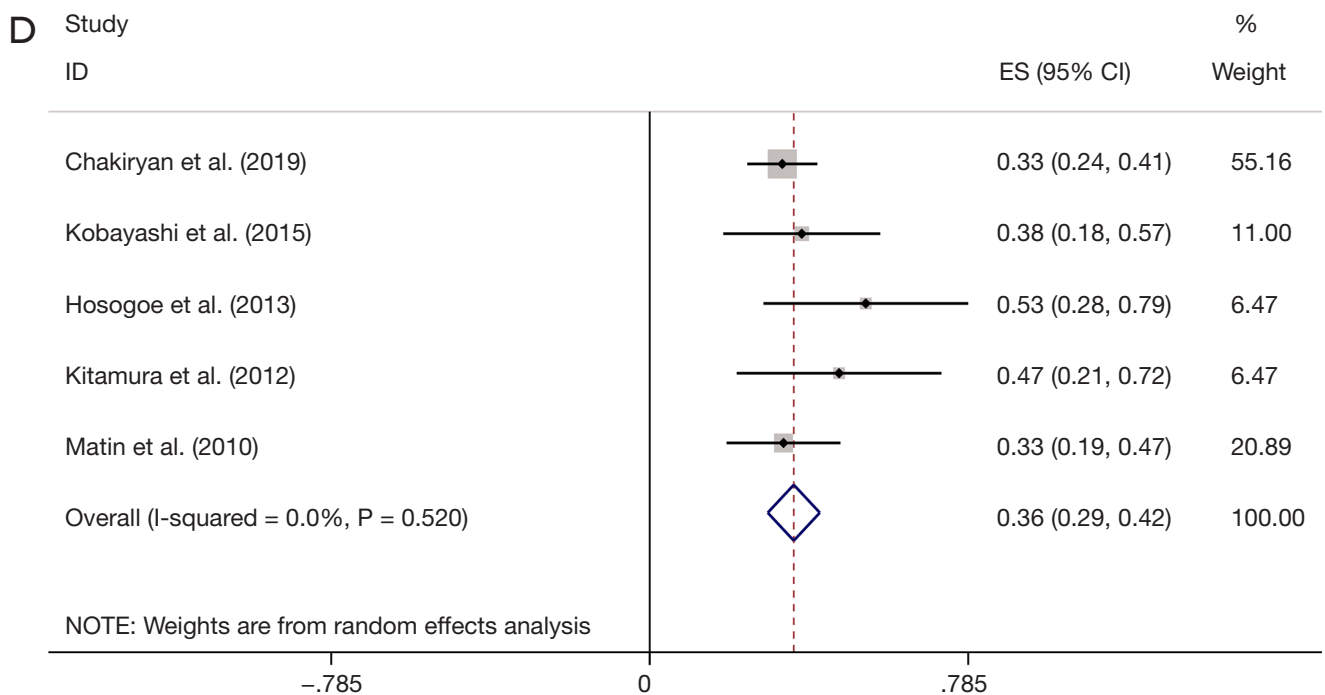

Figure 4 Subgroup analysis of pathological complete response (pCR) rate and pathological partial response (pPR) rate. (A) pCR rate of patients with stage TxN0; (B) pCR rate of patients with stage $\mathrm{TxNx}_{\mathrm{x}}$; (C) pPR rate of patients with stage TxN0; (D) pPR rate of patients with stage $\mathrm{TxNx}$.

rate among UTUC patients undergoing NAC followed by compared to patients subjected to surgery alone. Yang et al. in their study explored the effect of AC and NAC in UTUC patients, however, only two prognosis indicators of NAC (OS and CSS) were studied in this study (39). Studies published by Leow et al. and Gregg et al. included only two retrospective studies in their analyses. What's more, the above studies are largely heterogeneous in terms of patient characteristics, because they defined high-risk UTUC based on tumor grade, tumor burden, and architecture, rather than the TNM staging system $(5,40)$. Of note, Kim et al. in his studies included 4 articles and added more prognosis indicators (OS, CSS, PFS, and effect of NAC on downstaging) (17), but it evident that the included studies were uncomplete and all were based in one country, which perhaps cannot extend or apply to different ethnic groups. Therefore, evidence on the benefits and prognosis of NAC presented by these studies may not be persuasive. In our study, we included 19 articles and added 3 new effectivity indicators including DFS, pPR and pCR, which advanced the prognosis indicators and provided more meaningful and persuasive evidence to support the use of NAC in advanced UTUC patients.

Concerning $\mathrm{pPR}$ and $\mathrm{pCR}$, no previous pooled analyses have explored the 2 outcomes. In total, we included 12 studies, the pooled pCR was $11 \%$ (95\% CI: 0.07 , $0.14 ; \mathrm{P}=0.058)$, and pooled $\mathrm{pPR}$ rate was $40 \%$ (95\% CI:
$0.31-0.49 ; \mathrm{P}<0.001)$. To further assess the effect in various diseases and whether it lowers the heterogeneity, we conducted a subgroup analysis according to regional nodal metastasis (N0 or Nx). The pCR rate of patients with Stage TxN0 was $0.11(95 \%$ CI: $0.05-0.16 ; \mathrm{P}=0.014)$, whereas the pCR rate of patients with stage $\mathrm{TxNx}$ was $0.12(95 \% \mathrm{CI}$ : 0.03-0.21; $\mathrm{P}=0.191)$. Besides, stage TxN0 pPR rate was 0.27 (95\% CI: 0.23-0.31; $\mathrm{P}=0.339$ ), while stage $\mathrm{TxNx}$ pCR rate was 0.36 (95\% CI: 0.29-0.42; $\mathrm{P}=0.520$ ). Several possible reasons potentially led to high heterogeneity, including: (I) patients recorded in the included studies adopted different NAC regimens. (II) The number of UTUC patients in the present studies was small, and most studies included were retrospective. After analyzing these results, we found that NAC could improve the curative effect of advanced UTUC. Furthermore, due to the lack of published clinical trials findings on NAC effect in UTUC, this pooled analysis provided strong proof about this treatment for the clinicians.

In addition, the GRADE system was used to validate the accuracy of our findings. Given the methods we used, the pooled results could further validate the benefits of using NAC in advanced UTUC. What's more, findings from this study will provide insights to clinicians on the accurate use of the NAC regimen in managing UTUC patients. Besides, UTUC patients will appreciate better survival with NAC treatment. 
However, there were some limitations to this study. Firstly, most studies included were retrospective, this might have led to selection bias. Secondly, types, timing, cycle and disadvantages of using chemotherapy drugs were not included in subgroup analysis due to insufficient data from the included studies. Thirdly, survival endpoint was not clearly defined, and RNU was performed by different surgeons across different institutions and countries, this may influence the survival outcomes. To address these limitations and provide more reliable evidence on why NAC regimen should be adopted as a new treatment, there is a need for larger, more international, well-balanced, and multicenter prospective randomized studies or randomized control trials to demonstrate the actual effect of NAC when used to manage UTUC patients.

\section{Conclusions}

NAC treatment for patients with UTUC before RNU may provide better survival outcomes and achieve higher pathological response rate compared to when RNU is used independently. However, additional prospective randomized studies or randomized control trials should be undertaken to verify the benefits of NAC on prognosis in locally advanced UTUC patients are reliable.

\section{Acknowledgments}

We thank everyone who provided support for this study. We apologize for any omission of citations and references due to space limitation.

Funding: This work was supported by the National Natural Science Foundation of China (81902592), Hunan Province Key R\&D Program (2019SK2202) and Xiangya Hospital Youth Fund (2018Q09).

\section{Footnote}

Reporting Checklist: The authors have completed the PRISMA reporting checklist. Available at http://dx.doi. org/10.21037/tau-20-933

Peer Review File: Available at http://dx. doi. org/10. $21037 /$ tau-20-933

Conflicts of Interest: All authors have completed the ICMJE uniform disclosure form (available at http://dx. doi. org/10. 21037/tau-20-933). Dr. XZ served as an unpaid editorial board member of Translational Andrology and Urology from Mar 2015 to Feb 2021. The other authors have no conflicts of interest to declare.

Ethical Statement: The authors are accountable for all aspects of the work in ensuring that questions related to the accuracy or integrity of any part of the work are appropriately investigated and resolved.

Open Access Statement: This is an Open Access article distributed in accordance with the Creative Commons Attribution-NonCommercial-NoDerivs 4.0 International License (CC BY-NC-ND 4.0), which permits the noncommercial replication and distribution of the article with the strict proviso that no changes or edits are made and the original work is properly cited (including links to both the formal publication through the relevant DOI and the license). See: https://creativecommons.org/licenses/by-nc-nd/4.0/.

\section{References}

1. Roupret M, Babjuk M, Comperat E, et al. European Association of Urology Guidelines on Upper Urinary Tract Urothelial Cell Carcinoma: 2015 Update. Eur Urol 2015;68:868-79.

2. Siegel RL, Miller KD, Jemal A. Cancer statistics, 2020. CA Cancer J Clin 2020;70:7-30.

3. Margulis V, Shariat SF, Matin SF, et al. Outcomes of radical nephroureterectomy: a series from the Upper Tract Urothelial Carcinoma Collaboration. Cancer 2009;115:1224-33.

4. Yu J, Li G, Wang A, et al. Impact of squamous differentiation on intravesical recurrence and prognosis of patients with upper tract urothelial carcinoma. Ann Transl Med 2019;7:377.

5. Leow JJ, Martin-Doyle W, Fay AP, et al. A systematic review and meta-analysis of adjuvant and neoadjuvant chemotherapy for upper tract urothelial carcinoma. Eur Urol 2014;66:529-41.

6. Kang M, Jeong CW, Kwak C, et al. The characteristics of recurrent upper tract urothelial carcinoma after radical nephroureterectomy without bladder cuff excision. Yonsei Med J 2015;56:375-81.

7. Schinzari G, Monterisi S, Pierconti F, et al. Neoadjuvant Chemotherapy for Patients with Muscle-invasive Urothelial Bladder Cancer Candidates for Curative Surgery: A Prospective Clinical Trial Based on Cisplatin Feasibility. Anticancer Res 2017;37:6453-8. 
8. Margulis V, Puligandla M, Trabulsi EJ, et al. Phase II Trial of Neoadjuvant Systemic Chemotherapy Followed by Extirpative Surgery in Patients with High Grade Upper Tract Urothelial Carcinoma. J Urol 2020;203:690-8.

9. Leow JJ, Chong KT, Chang SL, et al. Upper tract urothelial carcinoma: a different disease entity in terms of management. ESMO Open 2017;1:e000126.

10. Tabayoyong W, Li R, Gao J, et al. Optimal Timing of Chemotherapy and Surgery in Patients with Muscle-Invasive Bladder Cancer and Upper Urinary Tract Urothelial Carcinoma. Urol Clin North Am 2018;45:155-67.

11. Xylinas E, Rink M, Margulis V, et al. Impact of renal function on eligibility for chemotherapy and survival in patients who have undergone radical nephro-ureterectomy. BJU Int 2013;112:453-61.

12. Birtle A, Johnson M, Chester J, et al. Adjuvant chemotherapy in upper tract urothelial carcinoma (the POUT trial): a phase 3, open-label, randomised controlled trial. Lancet 2020;395:1268-77.

13. Igawa $M$, Urakami S, Shiina $H$, et al. Neoadjuvant chemotherapy for locally advanced urothelial cancer of the upper urinary tract. Urol Int 1995;55:74-7.

14. Chen L, Ou Z, Wang R, et al. Neoadjuvant Chemotherapy Benefits Survival in High-Grade Upper Tract Urothelial Carcinoma: A Propensity Score-Based Analysis. Ann Surg Oncol 2020;27:1297-303.

15. Duplisea JJ, Petros FG, Li R, et al. Outcomes of nonmetastatic micropapillary variant upper tract urothelial carcinoma. Urol Oncol 2019;37:354.e19-26.

16. Rajput MZ, Kamat AM, Clavell-Hernandez J, et al. Perioperative outcomes of laparoscopic radical nephroureterectomy and regional lymphadenectomy in patients with upper urinary tract urothelial carcinoma after neoadjuvant chemotherapy. Urology 2011;78:61-7.

17. Kim DK, Lee JY, Kim JW, et al. Effect of neoadjuvant chemotherapy on locally advanced upper tract urothelial carcinoma: A systematic review and meta-analysis. Crit Rev Oncol Hematol 2019;135:59-65.

18. Pelcovits A, Mueller-Leonhard C, Mega A, et al. Outcomes of upper tract urothelial carcinoma with isolated lymph node involvement following surgical resection: implications for multi-modal management. World J Urol 2020;38:1243-52.

19. Meng X, Chao B, Vijay V, et al. High Response Rates to Neoadjuvant Chemotherapy in High-Grade Upper Tract Urothelial Carcinoma. Urology 2019;129:146-52 .

20. Chakiryan N, Martinez A, Gao L, et al. Optimizing the
Sequence of Chemotherapy for Upper Tract Urothelial Carcinoma with Clinically Positive Regional Lymph Nodes. J Urol 2019;202:76-82.

21. Hosogoe S, Hatakeyama S, Kusaka A, et al. Platinumbased Neoadjuvant Chemotherapy Improves Oncological Outcomes in Patients with Locally Advanced Upper Tract Urothelial Carcinoma. Eur Urol Focus 2018;4:946-53.

22. Kubota Y, Hatakeyama S, Tanaka T, et al. Oncological outcomes of neoadjuvant chemotherapy in patients with locally advanced upper tract urothelial carcinoma: a multicenter study. Oncotarget 2017;8:101500-8.

23. Kobayashi K, Saito T, Kitamura Y, et al. Effect of preoperative chemotherapy on survival of patients with upper urinary tract urothelial carcinoma clinically involving regional lymph nodes. Int J Urol 2016;23:153-8.

24. Porten S, Siefker-Radtke AO, Xiao L, et al. Neoadjuvant chemotherapy improves survival of patients with upper tract urothelial carcinoma. Cancer 2014;120:1794-9.

25. Kitamura H, Igarashi M, Tanaka T, et al. A role for preoperative systemic chemotherapy in node-positive upper tract urothelial carcinoma treated with radical nephroureterectomy. Jpn J Clin Oncol 2012;42:1192-6.

26. Cohen A, Kuchta K, Park S. Neoadjuvant and adjuvant chemotherapy use in upper tract urothelial carcinoma. Urol Oncol 2017;35:322-7.

27. Youssef RF, Shariat SF, Lotan Y, et al. Upper urinary tract urothelial carcinoma with loco-regional nodal metastases: insights from the Upper Tract Urothelial Carcinoma Collaboration. BJU Int 2011;108:1286-91.

28. Matin SF, Margulis V, Kamat A, et al. Incidence of downstaging and complete remission after neoadjuvant chemotherapy for high-risk upper tract transitional cell carcinoma. Cancer 2010;116:3127-34.

29. Almassi N, Gao T, Lee B, et al. Impact of Neoadjuvant Chemotherapy on Pathologic Response in Patients With Upper Tract Urothelial Carcinoma Undergoing Extirpative Surgery. Clin Genitourin Cancer 2018;16:e1237-42.

30. Liao RS, Gupta M, Schwen ZR, et al. Comparison of Pathological Stage in Patients Treated with and without Neoadjuvant Chemotherapy for High Risk Upper Tract Urothelial Carcinoma. J Urol 2018;200:68-73.

31. Martini A, Daza J, Poltiyelova E, et al. Pathological downstaging as a novel endpoint for the development of neoadjuvant chemotherapy for upper tract urothelial carcinoma. BJU Int 2019. doi: 10.1111/bju.14719.

32. Foerster B, Abufaraj M, Petros F, et al. Efficacy of Preoperative Chemotherapy for High Risk Upper Tract 
Urothelial Carcinoma. J Urol 2020;203:1101-8.

33. Green DA, Rink M, Xylinas E, et al. Urothelial carcinoma of the bladder and the upper tract: disparate twins. J Urol 2013;189:1214-21.

34. Rouprêt M, Babjuk M, Burger M, et al. European Association of Urology Guidelines on Upper Urinary Tract Urothelial Carcinoma: 2020 Update. Eur Urol 2020. doi: 10.1016/j.eururo.2020.05.042.

35. Seisen T, Jindal T, Karabon P, et al. Efficacy of Systemic Chemotherapy Plus Radical Nephroureterectomy for Metastatic Upper Tract Urothelial Carcinoma. Eur Urol 2017;71:714-8.

36. Roupret M, Babjuk M, Comperat E, et al. European Association of Urology Guidelines on Upper Urinary Tract Urothelial Carcinoma: 2017 Update. Eur Urol

Cite this article as: Qiu D, Hu J, He T, Li H, Hu J, Yi Z, Chen J, Zu X. Effect of neoadjuvant chemotherapy on locally advanced upper tract urothelial carcinoma: a pooled analysis. Transl Androl Urol 2020;9(5):2094-2106. doi:10.21037/tau-20-933
2018;73:111-22.

37. Spiess PE, Agarwal N, Bangs R, et al. Bladder Cancer, Version 5.2017, NCCN Clinical Practice Guidelines in Oncology. J Natl Compr Canc Netw 2017;15:1240-67.

38. Martini A, Falagario UG, Waingankar N, et al. Neoadjuvant versus adjuvant chemotherapy for upper tract urothelial carcinoma. Urol Oncol 2020;38:684.e9-684.e15.

39. Yang X, Li P, Deng X, et al. Perioperative treatments for resected upper tract urothelial carcinoma: a network metaanalysis. Oncotarget 2017;8:3568-80.

40. Gregg RW, Vera-Badillo FE, Booth CM, et al. Perioperative chemotherapy for urothelial carcinoma of the upper urinary tract: A systematic review and metaanalysis. Crit Rev Oncol Hematol 2018;128:58-64. 
Table S1 Detailed survival outcomes and efficiency rate of NAC in UTUC extracted from included studies

\begin{tabular}{|c|c|c|c|c|c|c|c|c|c|c|c|}
\hline \multirow{2}{*}{ Author } & \multirow{2}{*}{ Year } & \multicolumn{2}{|c|}{ os } & \multicolumn{2}{|c|}{ css } & \multicolumn{2}{|c|}{ PFS } & \multicolumn{2}{|c|}{ DSS } & \multirow{2}{*}{$\mathrm{pCR}$ rate } & \multirow{2}{*}{ pPR rate } \\
\hline & & $\mathrm{HR}$ & $95 \% \mathrm{Cl}$ & HR & $95 \% \mathrm{Cl}$ & $\mathrm{HR}$ & $95 \% \mathrm{Cl}$ & HR & $95 \% \mathrm{Cl}$ & & \\
\hline Foerster et al. & 2020 & NA & NA & NA & NA & NA & NA & NA & NA & 0.101 & 0.449 \\
\hline Margulis et al. & 2020 & NA & NA & NA & NA & NA & NA & NA & NA & 0.108 & NA \\
\hline Chakiryan et al. & 2019 & 2.56 & $1.72-4.00$ & NA & NA & NA & NA & NA & NA & NA & 0.327 \\
\hline Pelcovits et al. & 2020 & 1.85 & $1.30-2.63$ & NA & NA & NA & NA & NA & NA & NA & NA \\
\hline Meng et al. & 2019 & 8.01 & $0.94-67.85$ & NA & NA & 3.34 & $0.95-11.8$ & NA & NA & 0.08 & NA \\
\hline Martini et al. & 2019 & NA & NA & NA & NA & NA & NA & NA & NA & 0.15 & 0.21 \\
\hline Chen et al. & 2020 & 4.54 & $1.75-11.76$ & NA & NA & NA & NA & 3.43 & $1.25-9.47$ & NA & NA \\
\hline Liao et al. & 2018 & NA & NA & NA & NA & NA & NA & NA & NA & 0.094 & NA \\
\hline Almassi et al. & 2018 & NA & NA & NA & NA & NA & NA & NA & NA & 0.061 & 0.252 \\
\hline Hosogoe et al. & 2018 & 1.91 & $0.86-4.22$ & 1.97 & $0.87-4.46$ & 2.21 & $1.00-4.89$ & NA & NA & NA & 0.533 \\
\hline Kubota et al. & 2017 & 1.45 & $0.86-2.44$ & 2.08 & $1.13-3.84$ & 1.75 & $1.1-2.56$ & NA & NA & NA & NA \\
\hline Cohen et al. & 2017 & NA & NA & 1.64 & $0.93-2.94$ & NA & NA & NA & NA & NA & NA \\
\hline Kobayashi et al. & 2016 & 2.13 & $1.01-4.54$ & 2.94 & $1.49-5.88$ & 3.38 & $0.87-13.02$ & 5.2 & $1.49-18.10$ & NA & 0.375 \\
\hline Porten et al. & 2014 & 2.38 & $1.04-1.49$ & NA & NA & NA & NA & 6.75 & $1.90-24.04$ & NA & NA \\
\hline Kitamura et al. & 2012 & 3.85 & $1.29-11.1$ & NA & NA & NA & NA & NA & NA & 0.067 & 0.467 \\
\hline Youssef et al. & 2011 & NA & NA & NA & NA & NA & NA & 5.02 & $1.49-18.1$ & NA & NA \\
\hline Rajput et al. & 2011 & NA & NA & NA & NA & NA & NA & NA & NA & 0.15 & NA \\
\hline Matin et al. & 2010 & NA & NA & NA & NA & NA & NA & NA & NA & 0.21 & 0.33 \\
\hline Igawa et al. & 1995 & NA & NA & NA & NA & NA & NA & NA & NA & 0.13 & 0.4 \\
\hline
\end{tabular}

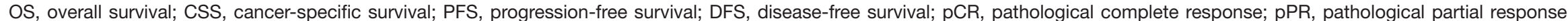
$\mathrm{NA}$, not available; HR, hazard ratio; $\mathrm{Cl}$, confidence interval.

Table S2 GRADE quality assessments of each pooled outcome

\begin{tabular}{|c|c|c|c|c|c|c|c|c|c|}
\hline \multirow{2}{*}{ Category } & \multicolumn{5}{|c|}{ Certainty assessment } & \multirow{2}{*}{$\begin{array}{l}\text { Effect relative }(95 \% \\
\qquad \mathrm{Cl})\end{array}$} & \multirow{2}{*}{$\begin{array}{l}\text { No. of participants } \\
\text { (studies) }\end{array}$} & \multirow{2}{*}{$\begin{array}{l}\text { Quality of the evidence } \\
\text { (GRADE) }\end{array}$} & \multirow{2}{*}{ Importance } \\
\hline & Study design & Risk of bias & Inconsistency & Indirectness & Imprecision & & & & \\
\hline OS & All observational studies & Serious & Not serious & Not serious & Serious & HR 2.18 (1.91-2.49) & 9 & $00 \bigcirc$ very low & CRITICAL \\
\hline CSS & All observational studies & Not serious & serious & Not serious & very serious & HR 1.99 (1.50-2.65) & 4 & 000 very low & CRITICAL \\
\hline PFS & All observational studies & Not serious & Not serious & serious & Very serious & HR 1.98 (1.48-2.66) & 4 & 000 very low & CRITICAL \\
\hline DFS & All observational studies & Not serious & Serious & Not serious & Very serious & HR 3.76 (2.16-6.56) & 4 & 000 very low & CRITICAL \\
\hline $\mathrm{pCR}$ & $\begin{array}{l}9 \text { observational studies; } \\
1 \text { clinical trail }\end{array}$ & Not serious & Not serious & Not serious & Serious & $0.11(0.07-0.14)$ & 10 & $\bigcirc \bigcirc \bigcirc \mathrm{low}$ & CRITICAL \\
\hline pPR & All observational studies & Serious & Not serious & Not serious & Serious & $0.40(0.31-0.49)$ & 10 & $00 \bigcirc$ very low & CRITICAL \\
\hline
\end{tabular}

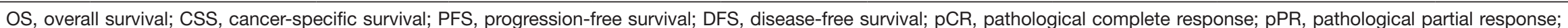
$\mathrm{HR}$, hazard ratio; $\mathrm{Cl}$, confidence interval. 
A

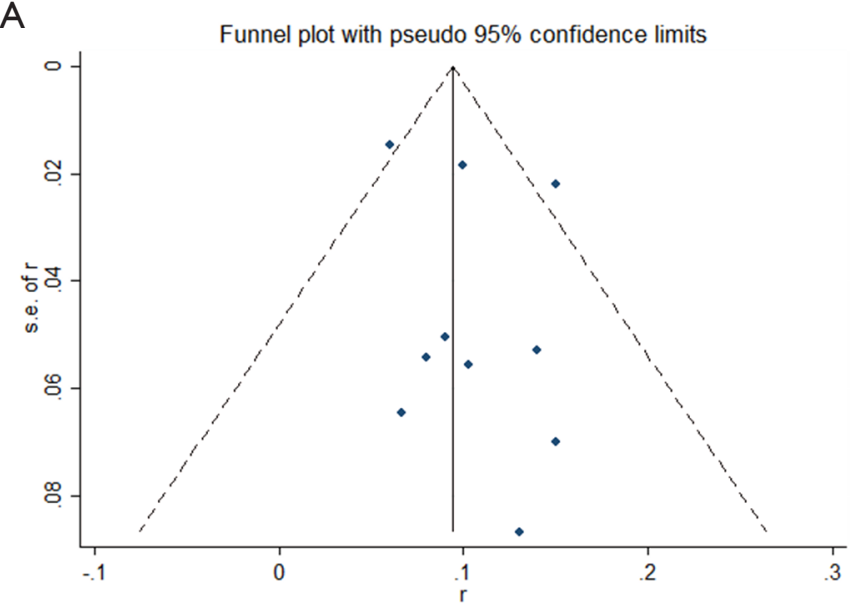

C

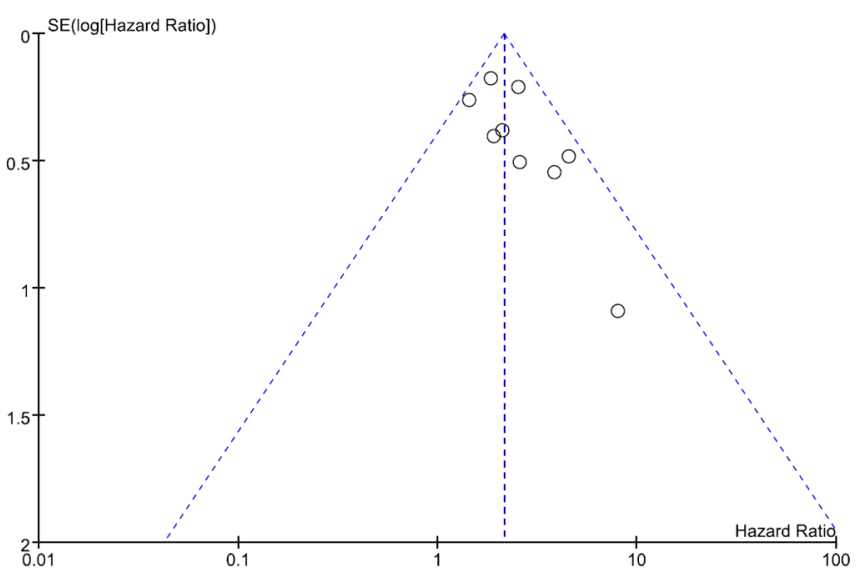

E

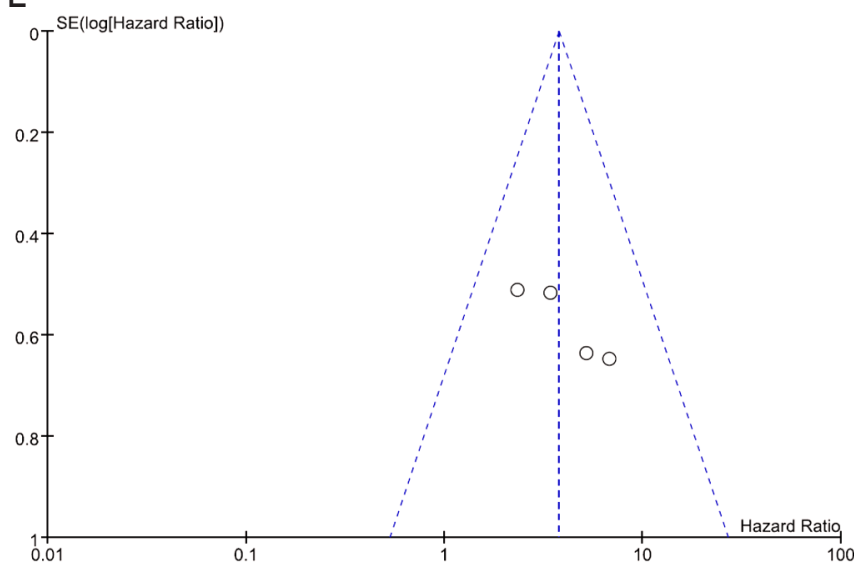

B

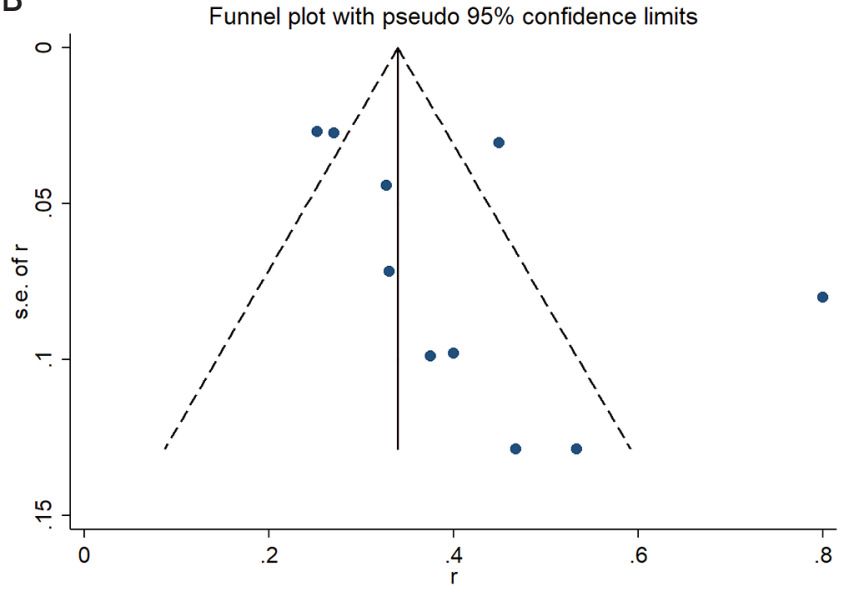

D

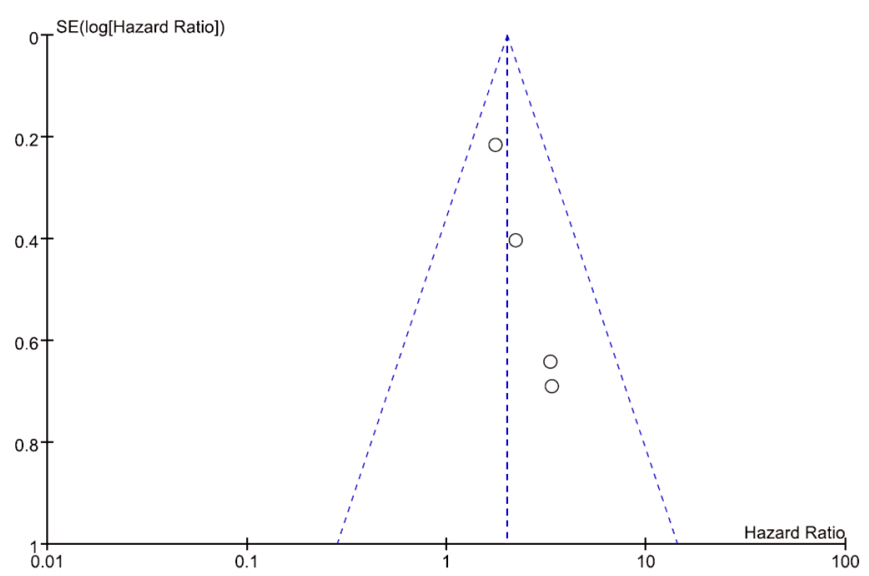

F

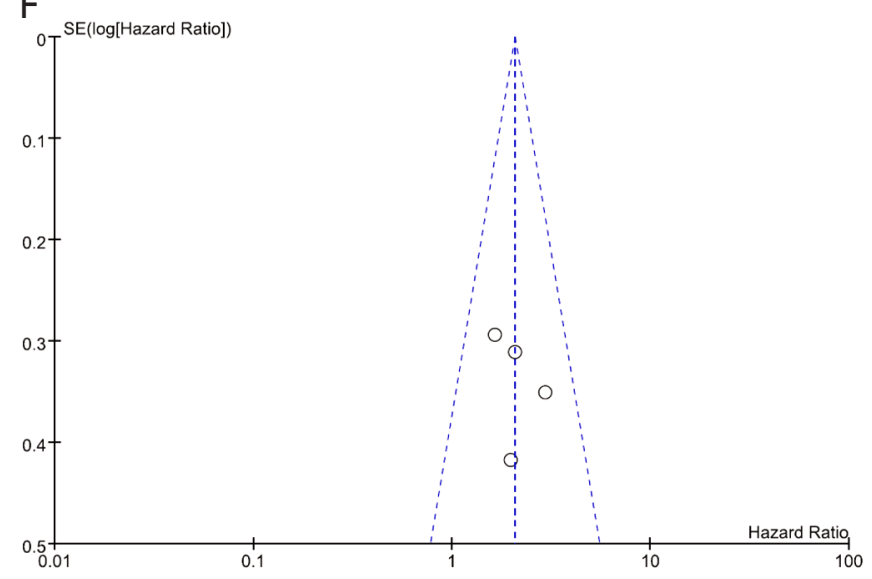

Figure S1 Funnel pots of each pooled survival outcomes and efficiency rates of NAC: (A) pathological complete response (pCR); (B) pathological partial response (pPR); (C) overall survival (OS); (D) progression-free survival (PFS). (E) disease-free survival (DFS); (F) cancer-specific survival (CSS). NAC, neoadjuvant chemotherapy. 
Table S3 Research status of clinical trials about NAC in UTUC

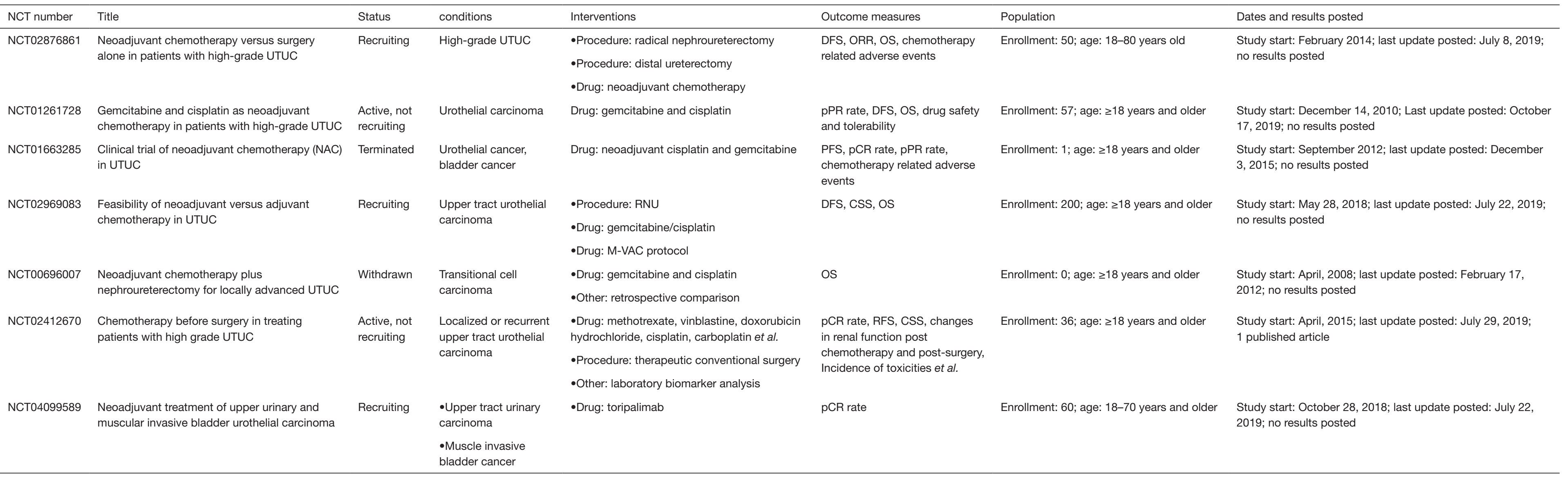

PFS, progression-free survival; DFS, disease-free survival; pCR, pathological complete response; pPR, pathological partial response; UTUC, upper tract urothelial carcinoma; OS, overall survival; CSS, cancer-specific survival. 